\title{
Feasibility and relevance of examining lymphoblastoid cell lines to study role of microRNAs in autism
}

\author{
Zohreh Talebizadeh, Merlin G. Butler, and Mariana F. Theodoro \\ Section of Medical Genetics and Molecular Medicine, Children's Mercy Hospitals and Clinics and \\ University of Missouri-Kansas City School of Medicine
}

\section{Scientific Abstract}

To assess the feasibility and relevance of using lymphoblastoid cell lines to study the role of noncoding RNAs in the etiology of autism, we evaluated global expression profiling of 470 mature human microRNAs from 6 subjects with autism compared with 6 matched controls. Differential expression (either higher or lower) for 9 of the 470 microRNAs was observed in our autism samples compared with controls. Potential target genes for these microRNAs were identified using computer tools which included several autism susceptibility genes. Our preliminary results indicate microRNAs should be considered and evaluated in the etiology of autism. In addition, analysis of this class of noncoding RNAs in lymphoblastoid cells has the potential to reveal at least a subset of brain-related microRNAs implicated in autism. Subsequently, this model system should allow for detection of complex subtle changes in susceptibility genes/pathways contributing to autism.

\section{Keywords}

microRNA; autism; lymphoblastoid cell lines; differential expression

\section{Introduction}

Autism (MIM 209850) is a genetically heterogeneous neurodevelopmental disorder which belongs to a group of conditions known as autism spectrum disorders (ASD) including classical autism, pervasive developmental disorder-not otherwise specified (PDD-NOS), and Asperger syndrome. Diagnostic features for classical autism include significant impairment in three developmental domains: reciprocal social behavior, language development, and repetitive/stereotypic behaviors (or a restricted range of interests) [Lord et al., 1994]. Linkage analysis has shown the involvement of at least 15 genes from different chromosomes [Yonan et al., 2003; Szatmari et al., 2007]. Despite the completion of several genome-wide linkage studies for autism, most of the loci identified have not been replicated in other studies. These inconsistent linkage results could be, in part, a reflection of the heterogeneous phenotype of autism.

It is estimated that $98 \%$ of the transcriptional output in humans and other mammals consists of noncoding RNAs (ncRNA) that do not code for protein but have other functions in cells [Mattick and Makunin, 2005]. Two main groups of ncRNAs include microRNAs and snoRNAs. microRNAs (miRNAs) are small RNA molecules of approximately 22 nucleotides, that regulate the expression of genes by binding to the 3 '-untranslated regions

Corresponding Author: Zohreh Talebizadeh, PhD, Children's Mercy Hospitals and Clinics, 2401 Gillham Road, Kansas City, MO 64108, Phone: (816) 983-6506, Fax: (816) 983-6501, Email: ztalebi@cmh.edu. 
(3'-UTR) of specific miRNAs [John et al., 2004; Farh et al., 2005] directing translational repression or transcript degradation. It is estimated that 10-30\% of human genes may be miRNA targets [John et al., 2004; Lewis et al., 2005]. miRNAs are processed from larger $(\sim 80 \mathrm{nt})$ precursors, the primary miRNA transcripts (pri-miRNAs), to a smaller hairpin precursor miRNA (pre-miRNA) which then yield mature 22-nucleotide miRNAs [Pillai, 2005]. Some miRNAs are within the introns of host genes and intronic miRNAs are usually expressed in coordination with their host gene mRNA [Baskerville and Bartel, 2005].

Like other RNAs, miRNA expression could be controlled at the posttranscriptional level [Pillai, 2005]. Some miRNAs are also subject to RNA editing, a process that, if occuring in the binding site, could change the target sites in the 3'-UTR regions [Blow et al., 2006; Kawahara et al., 2007]. RNA editing produces posttranscriptional modifications that result in sequence discrepancy between genomic DNA and its corresponding RNA. Recently, edited miR-376 was shown to silence a different set of genes compared with unedited form [Kawahara et al., 2007]. This finding indicates the critical role of RNA editing of miRNAs in the regulation of gene expression.

ncRNAs may provide the key to uncover links between neural development/function and neurological diseases [Mehler and Mattick, 2006]. Although, most are poorly characterized, recent discoveries indicate that ncRNAs may play important roles at various steps in the control of gene expression including RNA editing, RNA stability, and splicing [Vitali et al., 2003; Mattick and Makunin, 2005]. In addition, specific ncRNAs have been implicated in diseases such as various cancers and neurological disorders.

Multiple classes of ncRNAs are highly represented in the nervous system [Rogelj and Giese, 2004; Cao et al., 2006] emphasizing the likelihood that nervous system development and function is heavily dependent on RNA regulatory networks, and alterations of these networks may result in neurological diseases [Mehler and Mattick, 2006]. ncRNAs appear to regulate the maintenance of mature neural traits and synaptic plasticity [Krichevsky et al., 2003; Sempere et al., 2004; Conaco et al., 2006] and are heavily involved in synaptic function and memory formation [Martin and Kosik, 2002; Ashraf and Kunes, 2006; Ashraf et al., 2006; Schratt et al., 2006]. Hence, dysregulation of miRNAs have been reported in association with Alzheimer's disease [Krichevsky et al., 2003], X-linked mental retardation [Dostie et al., 2003], Parkinson's disease [Dostie et al., 2003], and Tourette's syndrome [Abelson et al., 2005]. In addition, Perkins et al. [2007] found that the ratio of microarray expression levels of miRNAs hosted in introns of coding genes versus mRNA of the host gene were significantly different for a group of miRNAs in patients with schizophrenia suggesting an altered biogenesis of miRNAs. Furthermore, bioinformatic tools have revealed the presence of a common motif in association with differentially expressed miRNA profiles in postmortem prefrontal cortex samples from schizophrenic patients [Perkins et al., 2007].

Despite a growing evidence for the regulatory influence of ncRNAs in gene expression, particularly in brain function, this group of regulatory factors has not been previously evaluated in autism spectrum disorders. Therefore, to assess the potential role of one class of small RNAs (e.g., miRNAs), we performed a pilot study examining global expression of mature miRNA profiling in growing lymphoblastoid cell lines (LCL) from subjects with autism compared with controls. Our study suggests that readily available LCL may be used to identify a subset of autism susceptibility miRNAs and potential target genes linked to biologically meaningful pathways which can be further studied in less accessible brain tissues. 


\section{Materials and Methods}

\section{Subjects}

Our autistic subject group (HI2879, HI1305, HI1704, HI2880, HI1319, and HI3430) consisted of three females (ages 6, 11, and 13 years old) and three males (ages 5, 12, and 14 years old) diagnosed with classical autism (one from simplex and five from multiplex families). The three autistic females had skewed X chromosome inactivation (i.e., ratio of > 80:20) using the androgen receptor gene polymorphism and were selected from our previous study on X chromosome inactivation patterns in females with autism [Talebizadeh et al., 2005]. Autistic and control samples were obtained from the Autism Genetics Resource Exchange (AGRE), a publicly available biomaterials repository located in Los Angeles, CA. The diagnosis of autism was established in the affected individuals with the use of the Autism Diagnostic Interview-Revised (ADI-R) [Lord et al., 1994]. Chromosome analysis and fragile $\mathrm{X}$ testing were reportedly normal. LCLs and clinical information (medical and pedigree data, diagnostic assessments and scores) were obtained on each subject from the AGRE. The control group consisted of six age and gender matched unaffected siblings from the AGRE families but unrelated to our selected autistic subjects.

\section{RNA Extraction}

Ten micrograms of total RNA was isolated from each cell line using the mirVana miRNA isolation kit (Ambion, Foster City, CA) to capture small RNAs. The high quality of the isolated RNA was confirmed through an Agilent Bioanalyzer system (Santa Clara, CA). The RNAs were then processed for a dual-channel $\mu$ Paraflo $^{\mathrm{TM}}$ microRNA microarray assay by LC Sciences (Houston, TX). Briefly, purified small RNAs were labeled with Cy3 (control) and Cy5 (autism) fluorescent dyes. The same RNA extraction stock was then utilized for PCR amplification as well as quantitative PCR, and DNA sequencing of selected miRNAs.

\section{microRNA Expression Profiling}

The $\mu$ Paraflo ${ }^{\mathrm{TM}}$ miRNA microarray assay was performed using a service provider (LC Sciences, Houston, TX). The assay required 4 to $8 \mu \mathrm{g}$ total RNA sample, which was size fractionated using a YM-100 Microcon centrifugal filter (Millipore, Billerica, MA) and the small RNAs (<300 nt) isolated were 3'-extended with a poly (A) tail using poly(A) polymerase. An oligonucleotide tag was then ligated to the poly (A) tail for later fluorescent dye staining; two different tags were used for the two RNA samples in dual-sample experiments. Hybridization was performed overnight on a $\mu$ Paraflo microfluidic chip using a micro-circulation pump (Atactic Technologies, Houston, TX) [Gao et al., 2004; Zhu et al., 2007]. On the microfluidic chip, each detection probe consisted of a chemically modified nucleotide coding segment complementary to target 470 microRNA from miRBase Sequence database version 9.0 (Sanger Institute, Cambridge, U.K.; http://microrna.sanger.ac.uk/sequences/) or multiple control probes (n=53) (e.g., PUC2PM-20B and PUC2MM-20B) and a spacer segment of polyethylene glycol to extend the coding segment away from the substrate. Internal control probes were used for quality control of chip production, sample labeling and assay conditions. The detection probes were made by in situ synthesis using photogenerated reagent chemistry. The hybridization melting temperatures were balanced by chemical modifications of the detection probes. Hybridization used $100 \mu \mathrm{L}$ 6xSSPE buffer $\left(0.90 \mathrm{M} \mathrm{NaCl}, 60 \mathrm{mM} \mathrm{Na}_{2} \mathrm{HPO}_{4}, 6 \mathrm{mM}\right.$ EDTA, $\mathrm{pH}$ 6.8) containing $25 \%$ formamide at $34{ }^{\circ} \mathrm{C}$. After RNA hybridization, tag-conjugating Cy3 and $\mathrm{Cy} 5$ dyes were circulated through the microfluidic chip for dye staining. Fluorescence images were collected using a GenePix 4000B laser scanner (Molecular Device, Sunnyvale, $\mathrm{CA}$ ) and digitized using Array-Pro image analysis software (Media Cybernetics, Bethesda, MD) [Gao et al., 2004; Zhu et al., 2007]. 


\section{Microarray Data Analysis}

Data analysis included the determination of detectable signals, calculation of signal intensities, and calculation of differential ratios ( $\log 2$ transformed). The data process began with background subtraction, Cy3/Cy5 channel normalization, detectivity determination, and then p-value calculation [Bolstad et al., 2003].

\section{Background Subtraction}

Background was determined using a regression-based background mapping method. The regression was performed on $5 \%$ to $25 \%$ of the lowest intensity data points excluding blank spots. Raw data matrix was then subtracted by the background matrix [Bolstad et al., 2003].

\section{Normalization}

Normalization was carried out using a LOWESS (Locally-weighted Regression) method on the background-subtracted data. The normalization removed system related variations, such as sample amount variations, different labeling dyes, and signal gain differences of scanners, so that biological variations could be readily identified [Bolstad et al., 2003].

\section{Detectivity Determination}

A transcript to be listed as detectable must meet at least two conditions: signal intensity higher than $3 \times$ (background standard deviation) and spot coefficient of variance $(\mathrm{CV})<0.5$. $\mathrm{CV}$ is calculated by (standard deviation)/(signal intensity). Six repeating probes were present on each chip for each specific target (i.e., miRNAs or controls) and a transcript was listed as detectable only if the signals from at least $50 \%$ of the repeating probes were above detection level [Bolstad et al., 2003].

\section{p-Value Calculation and Differentially Expressed miRNAs}

After the normalization, the p-values (t-test) of the difference between Cy3 and Cy5 signals were calculated using the values obtained after background subtraction and normalization and the standard deviations for $\mathrm{Cy} 3$ and $\mathrm{Cy} 5$ probe areas as previously described [Bolstad et al., 2003]. Fold change expression for differentially expressed probes was calculated using $\log 2$ transformed data.

Up to a maximum of 50 probes were identified as being significantly differentially expressed (i.e., p-value of less than 0.05) between autism and matched control subjects on each miRNA chip. The obtained lists of differentially expressed probes were compared among all six miRNA chips for concordance. Since we used data from a maximum of 50 probes to account for multiple tests, the calculated p-values were adjusted for 50 tests using the Bonferroni correction (i.e., p-value less than 0.001 ). To apply a more stringent Bonferroni adjustment, the $\mathrm{p}$-values need to be corrected for 150 tests (i.e., the total number of probes with a detectable expression on each chip) resulting in an adjusted p-value of less than 0.0003 . By applying such a stringent correction, the majority of the p-values for differentially expressed miRNAs in the autism group compared with controls still remained significant (i.e., p-value less than 0.0003).

\section{Prediction of Target mRNA}

Only a small number of target genes regulated by miRNAs have been experimentally verified. Thus, several computer programs have been designed using specific algorithms to predict target genes for miRNAs. Discovery of target genes have been complicated in humans; because, unlike the near-perfect complementarity between plant miRNA and their targets, the miRNA-mRNA binding in humans does not require a perfect sequence match. Therefore, it is expected that a rate of false positive predictions is associated with these 
computer programs. In general, miRNA target prediction methods are based on UTR sequence complementarity (miRNA-mRNA), free energy calculations of duplex formation, and degree of evolutionary conservation of target sequences.

Regulation by miRNAs is not a simple process. It is predicted that there are more than one target per miRNA, which in some cases can be grouped into functional categories [John et al., 2004]. On the other hand, more than one miRNA may target a specific gene [John et al., 2004]. Therefore, we used three publicly available programs: PicTar, TargetScan, and miRanda, for the prediction of target genes.

\section{Examination of RNA Editing}

Genomic DNA was extracted from available LCLs using the PureGene DNA isolation kit (Gentra Systems, Minneapolis, MN). One microgram of total RNA was utilized to synthesize cDNA by reverse transcription using Superscript III first-strand synthesis kit (Invitrogen, Carlsbad, CA). Human miR-92 has two predicted precursor sequences: miR-92a-1 on chromosome 13q31 and miR-92a-2 on Xq26. We used specific primers to amplify both copies of its pri-miRNA. Primer sequences for miR-146a, miR-132, miR-23a, miR-23b, and miR-320 were used as previously described by Blow et al. [2006]. Specific primers to amplify other differentially expressed pri-miRNAs were designed using the same criteria as following: miR-146b-F (5' AGAAGAAAGCATGCAAGAGCAGCG 3') and miR-146b-R (5' TGAAAGCTAAGTGGAGGCCGTACT 3') generating a 340-bp PCR product; miR-663-F (5' ATGGACGAGAATCACGAGCGATGT 3') and miR-663-R (5' TGCACCCTCCTGCATCAGTTTCTT 3') generating a 655-bp PCR product; miR-92-1-F (5' TGTCGCCCAATCAAACTGTCCTGT 3') and miR-92-1-R (5' TACTGCCAAATCTGACACGCAACC $3^{\prime}$ ) generating a 413-bp PCR product; and miRNAXq26-F (5' GCCAAAGGAAAGCCTGTTTCAGGA 3') and miRNA-Xq26-R (5' ACTGTAGCGCTGATCAGGAATGTC $3^{\prime}$ ) generating a 1106-bp PCR product.

Polymerase chain reactions (PCR) were performed in a total volume of $25 \mu \mathrm{l}$ using FailSafe PCR premix selection kit according to the manufacturer's instructions (Epicentre, Madison, WI). The amplified products were run on a $2 \%$ agarose gel, and the DNA bands were visualized by use of ethidium bromide staining under UV light. For sequence analysis, amplified products were purified using Performa DTR gel filtration cartridges (Edge BioSystems, Gaithersburg, MD). The purified PCR fragments were then sequenced using a capillary electrophoresis ABI 3100 Avant sequencer (Applied Biosystems, Foster City, CA).

Quantitative miRNA TaqMan Assay-Quantitative PCR (q-PCR) with the miRNA TaqMan assay developed by Applied Biosystems [Chen et al., 2005; Schmittgen et al., 2008] was performed by a service provider (Biopolymers facility at Harvard Medical School, Boston, MA). Each sample was run in quadruplicate. The quantitative reactions were normalized using an endogenous housekeeping gene, RNU24, as a control assay. Comparative $\mathrm{Ct}$ method was employed to quantify the real-time RT-PCR results. Change in expression between autism and control subjects with TaqMan assay was calculated according to Applied Biosystems guidelines. First, the average Ct cycle for the normalizer miRNA was subtracted from the average cycle for the test miRNA thus generating the Delta Ct. Then, the Delta Ct of the control subject was subtracted from the Delta Ct of its respective autism individual resulting in the Delta Delta $\mathrm{Ct}$, and fold change was finally calculated by $2^{- \text {(Delta Delta Ct) }}$.

\section{Results}

Two-color human microRNA array chip experiments were carried out commercially on RNA extracted from LCL. According to the hybridization data, a considerable number 
(31\%) of the tested miRNAs (about 150 out of 470 probes) had detectable expression. This compares with about $35 \%$ of miRNA detection rate reported in human brain samples using the same microarray platform [Rogaev, 2005]. Thus, our detection rate for LCL is very similar to brain tissue. Significant differential expressions (i.e., fold change greater than 1.5 at least in one comparison pair; $\mathrm{P}<0.05$ after a Bonferroni correction) were observed for 9 of 470 miRNAs in autism females compared with age-matched females in all 3 chips as shown in Table 1 and Figure1. Interestingly, there were several genes with neurological interest, in particular for autism, among the predicted targets for these 9 miRNAs (Table 2). Among the miRNAs identified with differential expression in autistic females, significantly different expression levels (in the same direction) were also found for the autistic males in 3 out of 3 comparisons for miR-132; in 2 out of 3 for miR-23a, miR-23b, miR-146b, miR-663, and miR-363; and in 1 out of 3 for miR-92 and miR-320.

Quantitative PCR validation with ABI TaqMan assay using mouse brain and mouse thymus RNA samples demonstrated excellent agreement between array data using LC Sciences microarray expression platform and ABI TaqMan assay (www.lcsciences.com; Www.appliedbiosystems.com). To further validate our microarray result, we performed ABI microRNA quantitative TaqMan assays [Chen et al., 2005; Schmittgen et al., 2008] for the 9 differentially expressed miRNAs except miR-663, since no specific ABI TaqMan assay has been developed for this miRNA. For miR-23b, miR-132, miR-146a, miR-146b, miR-92, miR-320, and miR-363, a significant differential expression, consistent with microarray findings, was confirmed for 4, 3, 6, 5, 5, 5, and 5 comparison sets (autism versus control), respectively. The TaqMan assay for miR-23a did not work. Interestingly, for each given miRNA, the highest fold change was seen for the same comparison set, according to both microarray and TaqMan assays. Furthermore, for the miR-363, a very high fold change (i.e., -73) was calculated using the array experiment for one comparison set (see F3 in Table 1).

Such a high degree of fold difference suggests the absence of expression in the relevant autism sample. The TaqMan result reinstated our expectation for this autism sample as its $\mathrm{Ct}$ value in the miR-363 assay was greater than 30 .

To evaluate the reproducibility of our data, we repeated microarray experiment for one control sample by dividing the extracted RNA into two aliquots and performing micoRNA array profiling on each replicate sample. Pair-wise Pearson correlation between the two replicates was calculated as $0.91(\mathrm{p}=0.00)$, suggesting a high level of reproducibility.

Genomic location of the identified differentially expressed miRNAs indicated that miR-363 and miR-92 are both located on the Xq26 chromosome band. Indeed, Xq26 contains a cluster of six miRNAs (miR-106a, miR-18b-, miR-20b, miR-19b-2, miR-92a-2, and miR-363) within about 850 nt on the human chromosome Xq (Figure 2). Examination of the expression level of the rest of the miRNAs from this Xq26 cluster showed either significant down-regulation or a trend in both male and female autistic subjects compared with controls with an exception of miR-363 and miR-92a-2 which were up-regulated in two autistic males.

To evaluate the presence of sequence discrepancy between genomic DNA and cDNA suggestive of RNA editing, we used specific primers for amplification of the detected differentially expressed miRNAs in our autistic samples as previously described by Blow et al. [2006]. With the exception of miR-23b which failed to amplify from cDNA, we were able to amplify these miRNAs from genomic DNA and cDNA in most of our subjects but did not detect any sequence discrepancy. 


\section{Discussion}

Autism is not a single entity but consists of a complex, heterogeneous neurobehavioral disorder with varying degrees of severity. To date, several candidate genes have been examined to evaluate their possible associations with autism. These candidate genes have generally been selected based on supportive linkage/cytogenetic evidence or the presence of certain findings at the clinical level in subjects with autism. The main technique in screening these candidate genes has been direct sequencing of gene exons using genomic DNA from subjects with autism compared with controls. Given that individuals with ASD are clinically heterogeneous, it is not surprising that most of the observed DNA sequence changes in candidate genes have not been reproduced in other studies. Furthermore, it is very likely that epigenetic factors (not identified by traditional genetic screening methods) play a role in the etiology of this complex disorder.

Autistic features are frequently seen in disorders with abnormal epigenetic factors: (1) Prader-Willi and Angelman syndromes are imprinting disorders involving the chromosome 15q11-q13 region containing brain specific snoRNAs; (2) Fragile X syndrome is caused by a loss of expression of FMRP, an RNA binding protein [Jin et al., 2004] that regulates the translation of its mRNA targets through the miRNA associated pathway [Jin et al., 2004; Duan and Jin, 2006]; and (3) Rett syndrome is caused by mutations in the MECP2 gene, a transcriptional repressor that can also act as a splicing regulator [Young et al., 2005]. Our recent reports for the involvement of epigenetic factors in autism (i.e., a higher degree of $\mathrm{X}$ inactivation skewness in autistic females [Talebizadeh et al., 2005] and alternative splicing of two X-linked neuroligin genes associated with autism [Talebizadeh et al., 2006]) further indicate a need for evaluation of the epigenetic factors in autism.

The study of posttranscriptional modifications in autism by evaluating ncRNAs (e.g., miRNAs) can provide a link between autism and epigenetic factors. Understanding the influence of ncRNAs on brain function and neurological characteristics in humans is at an early stage of research. Potential roles of ncRNAs have not been previously investigated in autism spectrum disorders. However, these small RNAs may play a significant role in brain function by the regulation of posttranscriptional modifications of target mRNAs. To assess the feasibility of utilizing LCL in neurological disorders, we performed global microarray expression techniques to evaluate overall expression profiling of 470 human miRNAs in subjects with autism compared with controls. In summary, although the sample size is small, our preliminary experiment indicates: (1) readily available LCL can be used for studying miRNA expression, and (2) significant differential expression for 9 of 470 miRNAs in autistic females compared with controls, one of which, miR-320, was also down-regulated in our autistic males.

Furthermore, several interesting results described below came from our study. These included the identification of autism susceptibility genes such as MECP2, FMR1, NLGN3, PTEN, EN2, as well as neurexin genes (NRXN1 and NRXN3) among the predicted targets for our differentially expressed miRNAs. Recently, implication of the SLITRK1 gene including a $3^{\prime}$-UTR mutation at the binding site for a unique miRNA has been reported in Tourette's syndrome (which has clinical overlap with ASDs) [Abelson et al., 2005]. There are at least six human Slitrk family proteins, neuronal transmembrane proteins controlling neurite outgrowth, with their gene expression mainly detected in the brain [Aruga et al., 2003]. We have observed that other members of the Slitrk family, SLITRK3, SLITRK5, and SLITRK6, are also among the potential targets for the miRNAs detected in our study. Two genes involved in apoptosis and autism (i.e., PTEN and TSC1) were also among the predicted target mRNAs for differentially expressed miRNAs in our autistic subjects. 
To identify if autism susceptibility genes are overrepresented among predicted target genes by the miRNAs differentially expressed in our autistic subjects, we reshuffled the list of tested miRNAs $(n=470)$ to produce a random set of 9 miRNAs. Similarly, predicted autism candidate gene targets were searched using this random group of 9 miRNAs. A total of 12 autism target genes were found in the random group (data not shown) compared with a total of 31 targets for the differentially expressed miRNAs (see Table 2). Furthermore, several of the genes listed in Table 2 (i.e., NLGN3, FMR1, PTEN, AUT2, SLITRK3, SLITRK4, EN2, SNRPN, NIPA1, and SNURF) were not seen among the predicted targets for our random set of 9 miRNAs. Our randomization analysis suggests that autism candidate genes are more overrepresented as targets amongst the observed differentially expressed miRNAs $(31 / 9=3.4)$ compared to a randomly selected set of miRNAs $(12 / 9=1.3)$.

A trend was observed for down regulation of six clustered miRNAs (i.e., miR-106a-363 cluster) located at chromosome Xq26 in our autistic subjects. Although, these miRNAs are separated only by a few nucleotides, their expression levels were not identical within each sample. In particular, miR-18b and miR-363 had the lowest expression level (less than ten times) compared to the rest of the miRNAs in this cluster for all the examined samples (autism and control) suggesting that while physically located in close vicinity, their expression might be controlled separately. The level of the miR-106a-363 cluster is substantially increased in human T-cell leukemia suggesting that the miR-106a-363 cluster might be oncogenic miRNAs [Lum et al., 2007]. An association of autism with oncogenes was further highlighted by a recent report of a genetic variant in the promoter region of the MET gene in autistic subjects suggesting a reduced MET gene expression in autism [Campbell et al., 2006].

It is interesting to note that miR-132 which is up-regulated in our autism subjects, is a brainrelated miRNA [Wu and Xie, 2006] with an abundant expression in the fetal hippocampus. Furthermore, its differential regulation along with other brain-related miRNAs has been suggested to play a role in Alzheimer's disease [Lukiw, 2007]. Moreover, two of the miRNAs from the Xq26 cluster, miR-92 and miR-20b, were recently reported to be expressed at a lower level in the prefrontal cortex of individuals with schizophrenia compared with unaffected subjects [Perkins et al., 2007].

Among the predicted target mRNAs are SNRPN, SNURF, and NIPA1 located at chromosome 15q11-q13, the Prader-Willi syndrome (PWS) imprinted region. The 15q11q13 chromosomal region contains imprinted loci including several brain-specific snoRNAs. One of these snoRNAs, HBII-52, exhibits a complementary sequence to a segment of serotonin $2 \mathrm{C}$ receptor (HTR2C) mRNA which undergoes both alternative splicing and RNA editing. HTR2C is among the predicted target genes for miR-23a and miR-23b. Alterations in the RNA editing of this serotonin receptor have been reported in patients with neurodevelopmental disorders including PWS [Kishore and Stamm, 2006]. An association between NIPA1 and behavioral outcomes in subjects with PWS has been suggested [Bittel et al., 2006]. Furthermore, frequent associations of autism with 15q11-q13 chromosomal abnormalities, make our observations more intriguing.

Autistic females examined in this study had a high degree of $\mathrm{X}$ inactivation skewness [Talebizadeh et al., 2005]. It will be interesting to determine whether any association is present with their extreme $\mathrm{X}$ inactivation pattern and miRNA expression profiling, particularly in the Xq26 oncogenic cluster. Comparison of the global miRNA expression between autistic females with and without such an extreme $\mathrm{X}$ inactivation pattern can provide valuable information, in particular, regarding the potential role of the $\mathrm{X}$ chromosome in gender discrepancy in autism. 
More recently, dysregulation of 28 miRNAs were reported in postmortem cerebellar cortex tissue of individuals with ASD compared with a control set of non-autistic cerebellar samples [Abu-Elneel et al., 2008]. The study design and data analysis used in that paper are not similar to that of our study; however, it is intriguing that 4 out of 9 miRNAs discussed in our study (i.e., miR-23a, miR-132, miR-146b, and miR-320) were among the list of 28 miRNAs showing dysregulation in autism brain samples compared with controls.

Overall, our study suggests that evaluation of miRNA expression profiling may have potential to identify pathways implicated in autism. However, additional research examining the expression of miRNAs at different developmental stages (prenatal and postnatal), cell types, and tissue sources will be necessary to clarify the roles miRNAs play in autism. Furthermore, evaluation of these ncRNAs in a subset of autistic subjects selected on the basis of the presence or absence of specific clinical or phenotypical features (e.g., level of cognition, language regression, etc.) will provide information on the role of miRNAs in relation to a specific biological function, which is underway in our laboratory.

Despite a growing number of discoveries emphasizing the importance of miRNAs on the etiology of human diseases during the last few years, there is a lack of sufficient experimental evidence identifying their corresponding mRNA targets. Experimental identification of miRNA targets is difficult because it requires development of specific criteria and knowledge base to determine miRNA targets which by itself depends upon the evaluation of an adequate number of functionally verified miRNA-mRNA targets. In a recent review article, Kuhn et al. [2008] outlined several methods by which to validate miRNA targets. A reporter system is recommended to asses whether or not the binding of a given miRNA to its specific mRNA target site will repress reporter protein production. Alternatively, miRNA-mRNA interaction may be validated in vivo by using antibodies against members of the Argonaute (Ago) protein family, a group of proteins known to bind to miRNAs with a partial degree of complementarity to the 3'-UTR of predicted target mRNAs, allowing for co-immunuprecipitation of Ago-bound mRNAs [Beitzinger et al., 2007; Easow et al., 2007].

The miRNA and its target mRNA must be co-expressed in order to repress the expression of its biological target. Co-expression may be examined by performing Northern blot analysis, quantitative real-time PCR, or in situ hybridization [Kuhn et al., 2008]. Modulation of miRNA concentration should correspond to a predictable change in the amount of protein encoded by the putative target mRNA measured by Western blot using a specific antibody against that protein, or other methods to quantify protein expression [Kuhn et al., 2008]. Utilizing antisense oligoribonucleotides can inhibit a specific endogeneous mature miRNA function and subsequently increase the expression of the target gene [Chen et al., 2006; Schratt et al., 2006]. Depending upon the protein target of interest, specific biological assays can be designed to evaluate the miRNA effect on target biological function (e.g., signaling pathways, cell proliferation, cell differentiation, cell death, etc.) [Kuhn et al., 2008]. Additionally, mouse miRNA knockout studies could be another valuable tool to investigate the functional impact of a given miRNA [Rodriguez et al., 2007; Thai et al., 2007].

While it is known that miRNAs regulate gene expression of target mRNAs through translation suppression or initiation of degradation of the mRNA, other functional aspects of this class of ncRNAs may be discovered. Recently, Vasudevan et al. [2007] reported that miRNAs may switch from repression to activation and can up-regulate translation in response to cell cycle stages, an important novel finding that should be considered in evaluating functional characterization of specific miRNAs. 
With the exception of a few genes, miRNA targets have been mainly identified based on bioinformatics prediction which requires experimental validation. Therefore, in the absence of a functional study, precaution should be taken in the interpretation of the predicted miRNA-mRNA pairs as some of the computer predictions may not have biological consequences. To detect the impact of specific miRNA misregulation on the expression level of their target genes, a comprehensive experimental design is needed to correlate miRNA and mRNA expression levels in a given sample, which is under consideration in our laboratory. Additional research is needed to identify the function of miRNAs and their interaction with gene networks to clarify the potential role they play in the etiology of ASDs.

Some miRNAs are tissue-specific while others are more abundant with a broader tissue distribution. Liang et al. [2007] evaluated miRNA expression profiling in 40 normal human tissues including brain which identified universally expressed miRNAs as well as exclusively or preferentially expressed miRNAs in certain tissue types. Our LCL expression profiling did not detect several miRNAs reported to be brain-specific or brain enriched such as mi-9, mi-9*, mi-124a, mi-135, mi-153, and mi-219 [Sempere et al., 2004]. However, expression of other brain-related miRNAs [Sempere et al., 2004; Schaefer et al., 2007] (i.e., miR-15a, miR-106a, miR-92, miR-132, miR-21, miR-23a, miR-27, miR-222, miR-125a, miR-125b, and miR-128) was detected in our study. Krichevsky et al. [2003] found significant changes in the expression of about 9 miRNAs during normal rat brain development, and expression of four of these miRNAs (i.e., miR-19b, miR-103, miR-125b, and miR-128) were detected in our study.

To evaluate the relation between brain and blood profiling for differentially expressed miRNAs found in our autism samples, we compared expression level of those miRNAs listed in Table 1 that were also examined by Liang et al. [2007]. According to their multiple human tissue profiling dataset, miR-23a, miR-92, and miR-320 were coordinately expressed in both brain and peripheral blood mononuclear cells (PBMC). Expressions of three other miRNAs (i.e., miR-146b, miR-132, and miR-23b) were also detected in both brain and PBMC but were 3,4 , and 34 times of what was detected in brain, respectively.

Compared with other organs, a highly diverse miRNA expression profile has been detected in both mouse and human brain [Miska et al., 2004; Sempere et al., 2004; Schaefer et al., 2007]. Using in situ hybridization data from the Allen Brain Atlas, Mercer et al. [2008] examined expression of ncRNAs in the mouse brain. Such a systematic analysis of the expressed ncRNAs revealed specific expression profiles associated with discrete functional sub-regions of brain, which was particularly apparent in the hippocampus, cerebral cortex, olfactory bulb, and cerebellum. For example, a number of ncRNAs were identified that exhibited expression profiles specific to four functionally distinct hippocampus sub-regions (i.e., the dentate gyrus, CA1, CA2, and CA3). Furthermore, a temporal and spatial expression profile of miRNAs has been reported in the developing and mature brain. Therefore, while it is more reasonable to evaluate miRNA expression in the implicated tissue (i.e., brain) for a neurodevelopmental disorder such as autism, wide diversity of miRNA expression profile reported for this highly complex organ and a paucity of sufficient autopsy brain samples make it very difficult to directly evaluate miRNAs in autistic brains.

Thus, it seems an enormous challenge to understand the role of miRNAs in the etiology of autism. Our study nevertheless represents an important first step in the possibility of utilizing LCL to identify at least a subset of miRNAs differentially expressed in autism subjects with a potential functional relevance. LCL are the most accessible source for molecular genetic studies, including those on autism. Baron et al. [2006] assessed the feasibility of using LCL for genome-wide expression profiling, and showed viability of this 
tool for identifying genes associated with autism. Their genome-wide analysis differentiated children with autism from typically developing children based upon observed differential expression of dopamine and serotonin related genes. Similar RNA source was utilized on a microarray expression profiling to evaluate autistic twins [Hu et al., 2006]. In this twin study, a gene expression difference that correlated with the severity of autism and language impairment was found in monozygotic twins of disparate severities. Furthermore, Baron and colleagues [2006] reported that gene expression profiling utilizing LCL differentiated subjects with autism and isodicentric chromosome 15 abnormalities from typically developing children. Genome-wide expression profiling of LCL also distinguished different forms of autism (i.e., due to a fragile X mutation or a 15q11-q13 duplication) and nonautistic controls [Nishimura et al., 2007]. These growing lines of evidence emphasize the value of utilizing LCL in autism gene expression studies as a surrogate in the absence of access to brain.

Herein, we assessed feasibility of LCL to study ncRNAs in autism. To begin exploring the possible roles for miRNAs in autism, we used a commercial microarray platform to characterize the expression of 470 miRNAs in LCL samples. Evaluating the expression level of miRNAs from LCL, a highly accessible non-brain tissue, has the potential to be highly scalable, allowing high-throughput analysis of at least a subset of brain-related miRNAs that are also expressed in LCL. This strategy offers an opportunity to take a first step in understanding the role of miRNAs and their target mRNAs in the etiology of autism.

Our pilot study indicates that a subset of brain-expressed miRNAs is also expressed in LCL. Therefore, in the absence of having access to a large number of autism brain samples, evaluating LCL samples would still be very informative in detecting changes for a subset of brain-expressed miRNAs. This tool will provide an opportunity to identify a subset of miRNAs and potential target mRNAs implicated in autism through posttranscriptional modification. Subsequently, more specific experiments can be applied on a limited number of available autism brain samples for further evaluation and confirmation of the preliminary findings in LCL samples.

\section{Acknowledgments}

We gratefully acknowledge the resources provided by the Autism Genetic Resource Exchange (AGRE) Consortium and the participating AGRE families. The Autism Genetic Resource Exchange is a program of Autism Speaks and is supported, in part, by grant 1U24MH081810 from the National Institute of Mental Health to Clara M. Lajonchere (PI). Partial funding support was provided from the Children's Mercy Hospital (CMH) Physician Scientist Award (01.3905) and the Cure Autism Now (CAN) Foundation (01.3956). This work is dedicated to the loving memory of Sedigheh Rezaian-Talebizadeh.

\section{Literature Cited}

Abelson JF, Kwan KY, O'Roak BJ, Baek DY, Stillman AA, Morgan TM, et al. Sequence variants in SLITRK1 are associated with Tourette's syndrome. Science. 2005; 310(5746):317-320. [PubMed: 16224024]

Abu-Elneel K, Liu T, Gazzaniga FS, Nishimura Y, Wall DP, Geschwind DH, et al. Heterogeneous dysregulation of microRNAs across the autism spectrum. Neurogenetics. 2008; 9(3):153-161. [PubMed: 18563458]

Aruga J, Yokota N, Mikoshiba K. Human SLITRK family genes: genomic organization and expression profiling in normal brain and brain tumor tissue. Gene. 2003; 315:87-94. [PubMed: 14557068]

Ashraf SI, Kunes S. A trace of silence: memory and microRNA at the synapse. Current Opinons in Neurobiology. 2006; 16(5):535-539.

Ashraf SI, McLoon AL, Sclarsic SM, Kunes S. Synaptic protein synthesis associated with memory is regulated by the RISC pathway in Drosophila. Cell. 2006; 124(1):191-205. [PubMed: 16413491] 
Baron CA, Tepper CG, Liu SY, Davis RR, Wang NJ, Schanen NC, et al. Genomic and functional profiling of duplicated chromosome 15 cell lines reveal regulatory alterations in UBE3A-associated ubiquitin-proteasome pathway processes. Human Molecular Genetics. 2006; 15(6):853-869. [PubMed: 16446308]

Baskerville S, Bartel DP. Microarray profiling of microRNAs reveals frequent coexpression with neighboring miRNAs and host genes. RNA. 2005; 11(3):241-247. [PubMed: 15701730]

Beitzinger M, Peters L, Zhu JY, Kremmer E, Meister G. Identification of human microRNA targets from isolated argonaute protein complexes. RNA Biology. 2007; 4(2):76-84. [PubMed: 17637574]

Bittel DC, Kibiryeva N, Butler MG. Expression of 4 genes between chromosome 15 breakpoints 1 and 2 and behavioral outcomes in Prader-Willi syndrome. Pediatrics. 2006; 118(4):e1276-1283. [PubMed: 16982806]

Blow MJ, Grocock RJ, van Dongen S, Enright AJ, Dicks E, Futreal PA, et al. RNA editing of human microRNAs. Genome Biology. 2006; 7(4):R27. [PubMed: 16594986]

Bolstad BM, Irizarry RA, Astrand M, Speed TP. A comparison of normalization methods for high density oligonucleotide array data based on variance and bias. Bioinformatics. 2003; 19(2):185193. [PubMed: 12538238]

Campbell DB, Sutcliffe JS, Ebert PJ, Militerni R, Bravaccio C, Trillo S, et al. A genetic variant that disrupts MET transcription is associated with autism. Proceedings of the National Academy of Science USA. 2006; 103(45):16834-16839.

Cao X, Yeo G, Muotri AR, Kuwabara T, Gage FH. Noncoding RNAs in the mammalian central nervous system. Annual Review of Neuroscience. 2006; 29:77-103.

Chen C, Ridzon DA, Broomer AJ, Zhou Z, Lee DH, Nguyen JT, et al. Real-time quantification of microRNAs by stem-loop RT-PCR. Nucleic Acids Research. 2005; 33(20):e179. [PubMed: 16314309]

Chen JF, Mandel EM, Thomson JM, Wu Q, Callis TE, Hammond SM, et al. The role of microRNA-1 and microRNA-133 in skeletal muscle proliferation and differentiation. Nature Genetics. 2006; 38(2):228-233. [PubMed: 16380711]

Conaco C, Otto S, Han JJ, Mandel G. Reciprocal actions of REST and a microRNA promote neuronal identity. Proceedings of the National Academy of Science USA. 2006; 103(7):2422-2427.

Dostie J, Mourelatos Z, Yang M, Sharma A, Dreyfuss G. Numerous microRNPs in neuronal cells containing novel microRNAs. RNA. 2003; 9(2):180-186. [PubMed: 12554860]

Duan R, Jin P. Identification of messenger RNAs and microRNAs associated with fragile X mental retardation protein. Methods in Molecular Biology. 2006; 342:267-276. [PubMed: 16957381]

Easow G, Teleman AA, Cohen SM. Isolation of microRNA targets by miRNP immunopurification. RNA. 2007; 13(8):1198-1204. [PubMed: 17592038]

Farh KK, Grimson A, Jan C, Lewis BP, Johnston WK, Lim LP, et al. The widespread impact of mammalian MicroRNAs on mRNA repression and evolution. Science. 2005; 310(5755):18171821. [PubMed: 16308420]

Gao X, Gulari E, Zhou X. In situ synthesis of oligonucleotide microarrays. Biopolymers. 2004; 73(5): 579-596. [PubMed: 15048782]

Hu VW, Frank BC, Heine S, Lee NH, Quackenbush J. Gene expression profiling of lymphoblastoid cell lines from monozygotic twins discordant in severity of autism reveals differential regulation of neurologically relevant genes. BMC Genomics. 2006; 7:118. [PubMed: 16709250]

Jin P, Alisch RS, Warren ST. RNA and microRNAs in fragile X mental retardation. Nature Cell Biology. 2004; 6(11):1048-1053.

Jin P, Zarnescu DC, Ceman S, Nakamoto M, Mowrey J, Jongens TA, et al. Biochemical and genetic interaction between the fragile $\mathrm{X}$ mental retardation protein and the microRNA pathway. Nature Neuroscience. 2004; 7(2):113-117.

John B, Enright AJ, Aravin A, Tuschl T, Sander C, Marks DS. Human MicroRNA targets. PLoS Biology. 2004; 2(11):e363. [PubMed: 15502875]

Kawahara Y, Zinshteyn B, Sethupathy P, Iizasa H, Hatzigeorgiou AG, Nishikura K. Redirection of silencing targets by adenosine-to-inosine editing of miRNAs. Science. 2007; 315(5815):11371140. [PubMed: 17322061] 
Kishore S, Stamm S. The snoRNA HBII-52 regulates alternative splicing of the serotonin receptor 2C. Science. 2006; 311(5758):230-232. [PubMed: 16357227]

Krichevsky AM, King KS, Donahue CP, Khrapko K, Kosik KS. A microRNA array reveals extensive regulation of microRNAs during brain development. RNA. 2003; 9(10):1274-1281. [PubMed: 13130141]

Kuhn DE, Martin MM, Feldman DS, Terry AV Jr, Nuovo GJ, Elton TS. Experimental validation of miRNA targets. Methods. 2008; 44(1):47-54. [PubMed: 18158132]

Lewis BP, Burge CB, Bartel DP. Conserved seed pairing, often flanked by adenosines, indicates that thousands of human genes are microRNA targets. Cell. 2005; 120(1):15-20. [PubMed: 15652477]

Liang Y, Ridzon D, Wong L, Chen C. Characterization of microRNA expression profiles in normal human tissues. BMC Genomics. 2007; 8:166. [PubMed: 17565689]

Lord C, Rutter M, Le Couteur A. Autism Diagnostic Interview-Revised: a revised version of a diagnostic interview for caregivers of individuals with possible pervasive developmental disorders. Journal of Autism and Developmental Disorders. 1994; 24(5):659-685. [PubMed: 7814313]

Lukiw WJ. Micro-RNA speciation in fetal, adult and Alzheimer's disease hippocampus. Neuroreport. 2007; 18(3):297-300. [PubMed: 17314675]

Lum AM, Wang BB, Li L, Channa N, Bartha G, Wabl M. Retroviral activation of the mir-106a microRNA cistron in T lymphoma. Retrovirology. 2007; 4:5. [PubMed: 17442096]

Martin KC, Kosik KS. Synaptic tagging -- who's it? Nature Reviews. Neuroscience. 2002; 3(10):813820. [PubMed: 12360325]

Mattick JS, Makunin IV. Small regulatory RNAs in mammals. Human Molecular Genetics. 2005; 14:R121-132. Spec No 1. [PubMed: 15809264]

Mehler MF, Mattick JS. Non-coding RNAs in the nervous system. The Journal of Physiology. 2006; 575(Pt 2):333-341. [PubMed: 16809366]

Mercer TR, Dinger ME, Sunkin SM, Mehler MF, Mattick JS. Specific expression of long noncoding RNAs in the mouse brain. Proceedings of the National Academy of Science USA. 2008; 105(2): 716-721.

Miska EA, Alvarez-Saavedra E, Townsend M, Yoshii A, Sestan N, Rakic P, et al. Microarray analysis of microRNA expression in the developing mammalian brain. Genome Biology. 2004; 5(9):R68. [PubMed: 15345052]

Nishimura Y, Martin CL, Vazquez-Lopez A, Spence SJ, Alvarez-Retuerto AI, Sigman M, et al. Genome-wide expression profiling of lymphoblastoid cell lines distinguishes different forms of autism and reveals shared pathways. Human Molecular Genetics. 2007; 16(14):1682-1698. [PubMed: 17519220]

Perkins DO, Jeffries CD, Jarskog LF, Thomson JM, Woods K, Newman MA, et al. microRNA expression in the prefrontal cortex of individuals with schizophrenia and schizoaffective disorder. Genome Biology. 2007; 8(2):R27. [PubMed: 17326821]

Pillai RS. MicroRNA function: multiple mechanisms for a tiny RNA? RNA. 2005; 11(12):1753-1761. [PubMed: 16314451]

Rodriguez A, Vigorito E, Clare S, Warren MV, Couttet P, Soond DR, et al. Requirement of bic/ microRNA-155 for normal immune function. Science. 2007; 316(5824):608-611. [PubMed: 17463290]

Rogaev EI. Small RNAs in human brain development and disorders. Biochemistry (Mosc). 2005; 70(12):1404-1407. [PubMed: 16417465]

Rogelj B, Giese KP. Expression and function of brain specific small RNAs. Reviews in the Neurosciences. 2004; 15(3):185-198. [PubMed: 15357141]

Schaefer A, O'Carroll D, Tan CL, Hillman D, Sugimori M, Llinas R, et al. Cerebellar neurodegeneration in the absence of microRNAs. The Journal of Experimental Medicine. 2007; 204(7):1553-1558. [PubMed: 17606634]

Schmittgen TD, Lee EJ, Jiang J, Sarkar A, Yang L, Elton TS, et al. Real-time PCR quantification of precursor and mature microRNA. Methods. 2008; 44(1):31-38. [PubMed: 18158130]

Schratt GM, Tuebing F, Nigh EA, Kane CG, Sabatini ME, Kiebler M, et al. A brain-specific microRNA regulates dendritic spine development. Nature. 2006; 439(7074):283-289. [PubMed: 16421561] 
Sempere LF, Freemantle S, Pitha-Rowe I, Moss E, Dmitrovsky E, Ambros V. Expression profiling of mammalian microRNAs uncovers a subset of brain-expressed microRNAs with possible roles in murine and human neuronal differentiation. Genome Biology. 2004; 5(3):R13. [PubMed: 15003116]

Szatmari P, Paterson AD, Zwaigenbaum L, Roberts W, Brian J, Liu XQ, et al. Mapping autism risk loci using genetic linkage and chromosomal rearrangements. Nature Genetics. 2007; 39(3):319328. [PubMed: 17322880]

Talebizadeh Z, Bittel DC, Veatch OJ, Kibiryeva N, Butler MG. Brief report: non-random X chromosome inactivation in females with autism. Journal of Autism and Developmental Disorders. 2005; 35(5):675-681. [PubMed: 16167093]

Talebizadeh Z, Lam DY, Theodoro MF, Bittel DC, Lushington GH, Butler MG. Novel splice isoforms for NLGN3 and NLGN4 with possible implications in autism. Journal of Medical Genetics. 2006; 43(5):e21. [PubMed: 16648374]

Thai TH, Calado DP, Casola S, Ansel KM, Xiao C, Xue Y, et al. Regulation of the germinal center response by microRNA-155. Science. 2007; 316(5824):604-608. [PubMed: 17463289]

Vasudevan S, Tong Y, Steitz JA. Switching from repression to activation: microRNAs can up-regulate translation. Science. 2007; 318(5858):1931-1934. [PubMed: 18048652]

Vitali P, Royo H, Seitz H, Bachellerie JP, Huttenhofer A, Cavaille J. Identification of 13 novel human modification guide RNAs. Nucleic Acids Research. 2003; 31(22):6543-6551. [PubMed: 14602913]

Wu J, Xie X. Comparative sequence analysis reveals an intricate network among REST, CREB and miRNA in mediating neuronal gene expression. Genome Biology. 2006; 7(9):R85. [PubMed: 17002790]

Yonan AL, Alarcon M, Cheng R, Magnusson PK, Spence SJ, Palmer AA, et al. A genomewide screen of 345 families for autism-susceptibility loci. American Journal of Human Genetics. 2003; 73(4): 886-897. [PubMed: 13680528]

Young JI, Hong EP, Castle JC, Crespo-Barreto J, Bowman AB, Rose MF, et al. Regulation of RNA splicing by the methylation-dependent transcriptional repressor methyl-CpG binding protein 2 . Proceedings of the National Academy of Science USA. 2005; 102(49):17551-17558.

Zhu Q, Hong A, Sheng N, Zhang X, Matejko A, Jun KY, et al. microParaflo biochip for nucleic acid and protein analysis. Methods in Molecular Biology. 2007; 382:287-312. [PubMed: 18220239] 


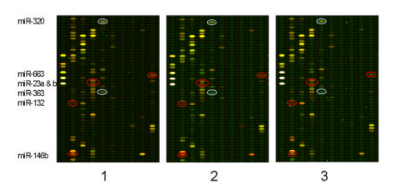

Figure 1.

Cy3/Cy5 ratio image of microRNA microarray expression. Representative microRNAs differentially expressed in 3 autism compared with 3 control females are marked with color code (red: up regulation and green: down regulation). Tissue source: lymphoblastoid cell lines. Pair sets: \#1 = 6 yr old autism/6 yr old control; \#2 = 11 yr old autism/12 yr old control; and \#3 = 13 yr old autism/13 yr old control. 


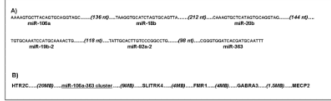

Figure 2.

microRNA cluster on human chromosome Xq26. A) Six microRNAs from the miR-106amiR-363 cluster (i.e., miR-106a, miR-18b, miR-20b, miR-19b-2, miR-92-2, and miR-363) and the number of base pair intervals with their subsequent microRNA are shown. B)

Genomic distances are indicated for several genes of interest for autism in close vicinity of the Xq26 microRNA cluster. 


\section{Table 2}

Selected predicted targets for microRNAs significantly differentially expressed in autism compared with control subjects. Selected predicted target genes related to autism are shown for each microRNA using PicTar, TargetScan, and miRanda programs.

\begin{tabular}{|lcl|}
\hline microRNA & Chromosome & Target genes related to autism \\
\hline microRNAs up-regulated in autistic compared with control subjects \\
\hline miR-23a & $19 \mathrm{p} 13$ & FMR1, PTEN, AUT2, NRXN1, NRXN3, HTR2C \\
miR-23b & $9 \mathrm{q} 22$ & FMR1, PTEN, AUT2, NRXN1, NRXN3, HTR2C \\
miR-132 & $17 \mathrm{p} 13$ & MECP2, PTEN, FMR1, HTR3A \\
miR-146a & $5 q 33$ & SLITRK3 \\
miR-146b & $10 \mathrm{q} 24$ & no predicted autism susceptibility target \\
miR-663 & $20 \mathrm{p} 11$ & no predicted autism susceptibility target \\
microRNAs down-regulated in autistic compared with control subjects \\
\hline miR-92 (a1-a2) & $13 q 31 ;$ Xq26 & FMR1, PTEN, TSC1, EN2 \\
miR-320 & $8 \mathrm{p} 21$ & MECP2, NLGN3, PTEN, AUTS2, TSC1, SLITRK3, SLITRK5, SNRPN \\
miR-363 & Xq26 & FMR1, TSC1 \\
additional miRNAs from the Xq26 cluster \\
\hline miR-106a & Xq26 & MECP2, PTEN, NIPA1, SLITRK3 \\
miR-18b & Xq26 & SNURF \\
miR-20b & Xq26 & MECP2, PTEN, NIPA1, SLITRK3 \\
miR-19b-2 & Xq26 & MECP2, PTEN, NIPA1, SLITRK6, FMR1, TSC1 \\
\hline
\end{tabular}

Selected predicted target genes related to autism (based on function and reported role) are shown for each microRNA using PicTar, TargetScan, and miRanda programs.

UTRL for PicTar: http://pictar.bio.nyu.edu/

UTRL for TargetScan: http://www.targetscan.org/

UTRL for miRanda: http://www.microrna.org/miranda_new.html 